\title{
Los aportes de Freire para repensar las prácticas de enseñanza con jóvenes y adultos
}

Fecha de recepción: 25 de febrero de 2020.

Fecha de aceptación: 20 de abril de 2020.

\section{Resumen}

En este artículo nos proponemos compartir resultados de investigaciones y su lectura desde los aportes de Paulo Freire para repensar la enseñanza en la modalidad de jóvenes y adultos. El trabajo se enmarca en el Proyecto "Prácticas educativas con jóvenes y adultos: políticas, sujetos y conocimiento", cuya investigadora responsable es María del Carmen Lorenzatti, con lugar de trabajo en el Área Educación, Centro de Investigaciones de la Facultad de Filosofía y Humanidades, Universidad Nacional de Córdoba (FONCYT/SECYT UNC). Nuestro objetivo es analizar los sujetos y el conocimiento en escuelas primarias, dando cuenta de corrimientos con relación a la enseñanza y el no reconocimiento de los estudiantes como sujetos cognoscentes. Compartimos reflexiones realizadas junto con docentes de la modalidad, sobre el lugar del conocimiento y señalamos una presencia naturalizada de clase que configuran procesos de enseñanza con los jóvenes y adultos. Finalmente, presentamos la potencialidad del trabajo con núcleos conceptuales, como propuesta que da centralidad a la tarea política pedagógica del docente. 


\title{
Freire's contributions to rethinking teaching practices with youth and adults
}

\begin{abstract}
In this article we propose to share research results and their reading from the contributions of Paulo Freire to rething teaching in the modality of young people and adults. The work is part of the Project "Educational practices with young people and adults: policies, subjects and knowledge", whose responsible researcher is Maria del C. Lorenzatti, with a place of work in the Education Area, Research Center of the Faculty of Philosophy and Humanities, National University of Córdoba (FONCYT/SECYT). Our goal is to analyze the people and knowledge in the elementary school, accounting for shifts in relation to teaching and non-recognition of students as cognitive subjects. We recover reflections about our workshops with teachers of the modality and we point out the naturalized presence of class that configure teaching processes with young people and adults. Finally, we present the potential of work with conceptual core, as a proposal that gives centrality to the politics and pedagogical task of the teacher.
\end{abstract}

Keywords: subjects; knowledge; youth and adults; awareness.

\section{Introducción}

Revisitar la pedagogía de Paulo Freire nos ubica, tal como señala Rodríguez (2015), en las disputas por trascender el mito y transitar una relectura crítica y situada en las condiciones de producción que plantean las políticas neoliberales actuales en América Latina.

A lo largo del trabajo sostenido con educadores de jóvenes y adultos hemos identificado algunas formas de enseñanza en las escuelas primarias y consideramos que los aportes del pedagogo brasilero ofrecen herramientas fértiles para discutir la enseñanza en la modalidad. Estas prácticas se han configurado en un proceso histórico que fue tallando la conformación de la modalidad en la Argentina. Estos procesos, no exentos de luchas, han dejado huellas palpables en las instituciones y en las prácticas de enseñanza.

En trabajos anteriores (Bowman, 2011; Lorenzatti, 2018) mostramos evidencias que nos permiten hablar de procesos de corrimiento de la enseñanza en aulas de nivel primario de jóvenes y adultos. Este fenómeno puede responder, en principio, a dos situaciones que no son dicotómicas sino complementarias. Por un lado, la realidad de los sujetos que asisten a la modalidad interpela fuertemente a los maestros y los lleva, en muchos casos, a desarrollar tareas que exceden la enseñanza y pertenecen al campo psicológico o del trabajo social. Por otro lado, identificamos, desde hace tiempo, una manifestación recurrente en los docentes de la modalidad y es la que refiere que "para enseñar en la modalidad hay que partir de lo que los alumnos saben". Lorenzatti (2018) muestra que hay circulación de conocimiento cotidiano y que en pocas ocasiones se realiza un abordaje curricular de las distintas disciplinas favoreciendo procesos de apropiación de otros conocimientos. De esta manera, se desdibuja la articulación entre los saberes de los estudiantes y los escolares, que son reconocidos como saberes socialmente válidos por los sujetos adultos.

En este artículo presentamos reflexiones sobre los aportes de Freire para pensar la enseñanza en las escuelas de jóvenes y adultos. Para ello, analizamos dos categorías 
de este autor brasilero: sujetos (estudiantes y docentes) y conocimientos (cotidianos y escolares). Estas construcciones teóricas ofrecen herramientas para tensionar los saberes que los adultos aportan al espacio áulico y la enseñanza que desarrollan los maestros.

En primer lugar, presentamos reflexiones construidas desde nuestras investigaciones donde analizamos tanto a estudiantes como educadores de la modalidad de Educación Permanente de Jóvenes y Adultos (en adelante EPJA) con relación al conocimiento. En segundo lugar, profundizamos la categoría conocimiento y su lugar en situaciones educativas con jóvenes y adultos. Interesa analizar las intersecciones entre enseñar reconociendo los saberes que circulan, lo que establece el currículum y el lugar de los estudiantes en la situación educativa. Finalmente, proponemos algunas reflexiones en torno al conocimiento, los sujetos y la especificidad de la enseñanza en EPJA en la construcción de una propuesta centrada en núcleos conceptuales.

\section{Sujetos y conocimientos en las escuelas de jóvenes y adultos}

Las experiencias educativas con jóvenes y adultos se encuentran atravesadas por múltiples heterogeneidades que comprenden instituciones, sujetos y prácticas. A lo largo de la historia identificamos políticas que se plasmaron en diferentes modos institucionales y, a la vez, encontramos trayectorias socioeducativas diversas tanto en los estudiantes como en los educadores. Estas trayectorias de los sujetos participantes generan también diferentes prácticas educativas, es decir la producción de singularidades en el trabajo con el conocimiento y, de esta manera, en la construcción de sentidos que este asume para los sujetos.

En nuestros estudios no solo observamos saberes que poseen jóvenes y adultos sino también el "no reconocimiento" que realizan de los mismos, es decir que ellos menosprecian sus conocimientos cotidianos y asumen como legítimos los conocimientos escolares. Esto sucede, especialmente, con el conocimiento de la cultura escrita. Dichiara (2018) estudió la trayectoria de una mujer que aprendió a leer y escribir con miembros de la iglesia Testigos de Jehová en su casa, pero sintió la necesidad de acudir a la escuela de adultos "para aprender bien". Lorenzatti y Arrieta (2017) muestran resultados similares en relación con este modo escritural formal que se asocia a otro contenido de enseñanza buscado por los estudiantes en el espacio escolar: "la buena ortografía" que vinculan a la prolijidad y la "linda letra". Son estas prácticas de escritura que demandan a la institución escolar porque es el mandato fundacional de la escuela y de la escuela de adultos específicamente. De esta manera, la escuela es el espacio legitimado en la transmisión del conocimiento. Freire, en Pedagogía del oprimido, sostiene que en los adultos se produce una "autodesvalorización" y que se asumen como propios "... los criterios del saber que les son impuestos" (2000: 64).

También identificamos los saberes que adultos no escolarizados tienen en relación con la cultura escrita: conocen una serie de "papeles", documentos, formularios, tarjetas, cartas, almanaques, documentos religiosos, cancioneros, boletas de impuestos que conforman su "pequeño mundo" (Heller, 1997). A su vez, lo cotidiano de estas personas incluye acciones en instituciones que demandan otras prácticas, que exigen otros conocimientos donde siempre está involucrada la cultura escrita. Las políticas sociales y la burocracia del sistema administrativo ingresan a la vida de estos adultos y les exigen relacionarse con objetos escritos, no solo a partir de los papeles mencionados sino también a través de la pantalla de la computadora que exhibe información válida para la vida de estas personas (Lorenzatti, 2018).

Estos estudios, desarrollados desde la perspectiva de los nuevos estudios de literacidad (Street, 1984, 2009; Kalman, 2004; Zavala et al., 2004; Kalman y Street, 2009) permiten 
ser analizados también desde la perspectiva freireana. De manera particular, desde la categoría sujeto, cuando Freire se refiere a la población nordestina en la década de 1960, en Educación como práctica de la libertad (1976), sostiene que alfabetizandos y alfabetizadores son sujetos cognoscentes que no están apenas en el mundo sino con él y traban una relación dialógica en torno al objeto de conocimiento que es su realidad circundante. De esta forma, crean los conocimientos a partir de sus propias experiencias y reflexiones sobre su vida cotidiana. En esa relación dialógica con el contexto, con su realidad circundante, los sujetos construyen sus conocimientos, y eso lo significa una estudiante adulta cuando reflexiona sobre su condición de no alfabetizada expresando "como que nací conociendo". Este modo de reconocerse muestra también un sujeto creador que anula la visión del proceso de alfabetización como la "pura enseñanza de la palabra, de las sílabas o de las letras" (Freire, 2001: 104) sino que se convierte en un acto político y en un acto de conocimiento y desde allí posiciona al alfabetizando como un sujeto creador.

Sin embargo, nuestras observaciones en escuelas y centros de alfabetización denuncian cierta ausencia de estos conocimientos sociales en los procesos de enseñanza. Se recurre muchas veces al silabeo y deletreo apelando a la palabra generadora desligada del análisis de la realidad. Sucede lo que el educador brasilero sostiene cuando se lee "El ala es del ave, Eva vio la uva...", se reproducen prácticas desconociendo que se trata de "contextos lingüísticos que, al ser mecánicamente memorizados y repetidos, se ven desprovistos de su auténtica dimensión como pensamiento-lenguaje, en su dinámica relación con la realidad" (Freire, 1975: 24).

Los educadores manifiestan, en los talleres realizados, ${ }^{1}$ que no están preparados para trabajar con grupos de estudiantes adultos porque su formación inicial se orienta hacia la enseñanza de niños. Históricamente la formación docente ha contemplado de forma intermitente algunas políticas destinadas a la preparación para el trabajo con adultos. A partir de 2008, se promueve a nivel nacional una reforma de la formación docente, se realiza una reforma curricular referida a la formación docente inicial y se incorpora, de modo optativo, la posibilidad de dictar tres seminarios sobre las modalidades educativas que reconoce la Ley Nacional de Educación (No 26.206/2006). Una de ellas, es la educación de jóvenes y adultos. ${ }^{2}$ Sin embargo, con estos avances persisten algunas huellas de las matrices fundantes y se observan dificultades en la construcción de una visión que integre estos seminarios al resto de las disciplinas.

En un estudio regional con universidades e institutos de formación docente de Brasil, Paraguay, Uruguay y la Argentina, ${ }^{3}$ realizado entre 2014 y 2015, se analizó la legislación y la normativa general en materia de formación docente inicial de educación de jóvenes y adultos y educación rural y su puesta en práctica en las instituciones participantes. En los cuatro países estudiados la problemática emerge como área de vacancia en las políticas de formación docente inicial, aunque se observa mayor presencia curricular de manera optativa en la Argentina y Brasil. Se observa también, en el caso de nuestro país, un grado de desconocimiento de las modalidades (y de manera particular, EPJA) en profesores de distintos espacios curriculares del profesorado de nivel primario. En esta línea, se identifica la ausencia de un abordaje didáctico específico en los seminarios disciplinares de la orientación de educación de jóvenes y adultos (Lorenzatti, 2016).

1 Talleres con docentes de los Centros Educativos de Jóvenes y Adultos, dependientes de la Municipalidad de la ciudad de Córdoba, realizados entre 2017 y 2019. Esta propuesta se desarrolla en el marco de una actividad de extensión.

2 En la provincia de Córdoba, esta reforma se puso en práctica en 2009. Los seminarios se implementaron hasta 2015, cuando se redujo la propuesta a un solo espacio curricular, porque los dos restantes fueron reemplazados por la enseñanza de inglés, vinculada a la propuesta de extensión de la jornada.

3 Proyecto “Estudio de la situación de las políticas de formación docente para educación de jóvenes y adultos y educación rural en países del Mercosur". "Programa de Apoyo al Sector Educativo del MERCOSUR (PASEM)". Coordinado desde la Prosecretaría de Relaciones Internacionales, Universidad Nacional de Córdoba, Argentina. 
Estos resultados nos interpelan para repensar las prácticas educativas con los jóvenes y adultos. Por un lado, la necesidad de que los educandos puedan asumirse como sujetos cognoscentes. Freire sostiene en Pedagogía de la esperanza. Un reencuentro con Pedagogía del Oprimido:

En el fondo, lo que quiero decir es que el educando se torna realmente educando cuando y en la medida en que conoce o va conociendo los contenidos, los objetos cognoscibles, y no en la medida en que el educador va depositando en él la descripción de los objetos, o de los contenidos. El educando se reconoce conociendo los objetos, descubriendo que es capaz de conocer, asistiendo a la inmersión de los significados en cuyo proceso se va tornando también significador crítico. (2002: 66)

En el mismo texto, insiste también en la importancia del docente, cuando señala que el educador "esté al tanto de la comprensión que tiene el pueblo" (2002: 44). Freire autorreferencia esta situación en su experiencia con campesinos:

Fue un aprendizaje largo, que implicó un recorrido, no siempre fácil, casi siempre sufrido, hasta que me convencí de que aun cuando estaba seguro de mi tesis, de mi propuesta, y no tenía ninguna duda respecto a ellas, era imperioso, primero, saber si coincidían con la lectura del mundo de los grupos o de la clase social a quienes me dirigía; segundo, se me imponía estar más o menos familiarizado con su lectura del mundo, puesto que solo a partir del saber contenido en ella, explícito o implícito, podría discutir mi lectura del mundo, que igualmente guarda y se funda en otro tipo de saber. (2002: 41)

Estos reconocimientos, que son sustanciales en el vínculo pedagógico propuesto por Freire, requieren ser problematizados, entendiendo que son producto de las situaciones de vida de los estudiantes jóvenes y adultos. Es decir, la lectura del mundo que realizan los sujetos deviene de esquemas de percepción que se construyen a partir de condiciones sociales concretas. Como sostiene Heller (1977) los sujetos necesitan aprender a "usar" las cosas, apropiarse del lenguaje, de las costumbres para conservarse en una época determinada en el ámbito de un estrato social dado.

Desde estos posicionamientos, hay que avanzar en la reconceptualización de los jóvenes y adultos y sus vínculos con los conocimientos. Para ello es necesario realizar un trabajo en terreno, averiguar con ellos, desde sus propias prácticas, percepciones y representaciones qué necesitan y qué creen que necesitan. En la condición de trabajadores, desempleados, padres, hermanos, es importante indagar cuáles son sus aspiraciones y frustraciones como ciudadanos y también atender a la significación que cobran sus experiencias vitales para la construcción y/o recuperación de una identidad como sujeto social. Es decir, recuperar los saberes propios de la experiencia vivida, tal como plantea Freire en la Novena Carta (2002b) y a partir de allí discutir y plantear propuestas de enseñanza.

\section{La enseñanza en EPJA: entre el currículum y enseñar desde lo que saben}

En nuestras investigaciones y proyectos extensionistas con educadores de jóvenes y adultos se evidencia la necesidad de profundizar el trabajo sobre las decisiones que toman los docentes en relación con su práctica de enseñanza. En tal sentido, los últimos libros de Freire proponen el análisis crítico de la práctica docente con relación a los conocimientos y a los estudiantes en las escuelas. El autor profundiza particularmente en la posición ético-política, asumiendo la no neutralidad de la educación y 
la visibilización de las situaciones existenciales de los sujetos, estudiantes y docentes. Freire analiza de qué manera esas condiciones configuran las relaciones sociales en el vínculo pedagógico.

Freire aporta herramientas conceptuales para pensar cómo se construyen las relaciones con el conocimiento a partir del "contexto concreto" donde viven los estudiantes. Sostiene que pensar la práctica implica conocer cómo piensan los estudiantes en su contexto real, en su vida cotidiana; conocer qué saben independientemente de la escuela y conocer las condiciones materiales desfavorables de vida (2002b). En esta línea se entiende que la lectura del mundo no es absoluta y está construida desde una particular posición que moldea esa relación con el mundo. Además de estas condiciones concretas, el autor brasilero insiste en la idea de "que los oprimidos hospedan al opresor" (2002b: 36). Más precisamente, sostiene que han incorporado los esquemas de percepción de los opresores. Esta afirmación se aplica no solo a los estudiantes, entendidos como sujetos excluidos, sino también a los docentes ya que se forman en categorías pedagógicas que excluyen el estudio de la educación de jóvenes y adultos, que invisibilizan la relación de la modalidad con el sistema educativo y la especificidad pedagógica de este campo. Esos modos de formación se expresan y articulan en las propuestas de enseñanza y en maneras de construir al sujeto del aprendizaje.

Estos señalamientos invitan a pensar las prácticas docentes como prácticas sociales, complejas, situadas, históricas y configuradas a partir de sujetos educadores, estudiantes y sus contextos de desarrollo. Las heterogeneidades que construyen los espacios institucionales de las escuelas de jóvenes y adultos producen singularidades en el trabajo con el conocimiento y, de esta manera, en el sentido que este asume para los sujetos.

En este marco, recuperamos los decires de Freire en Política y Educación (2001a) cuando propone la importancia del conocimiento de la vida cotidiana de los sujetos y sostiene la necesidad de construir una comprensión crítica de la cotidianeidad del medio popular por parte de los educadores. En esta línea, enfatiza que “...educadores y educadoras no pueden pensar únicamente en los procedimientos didácticos y los contenidos que se han de enseñar a los grupos populares. Los propios contenidos que se han de enseñar no pueden ser extraños a esa cotidianeidad" (2001a: 31).

En Cartas a Cristina. Reflexiones sobre mi vida y mi trabajo (1996), Freire relata de qué manera fue construyendo ese conocimiento de su entorno concreto inmediato, buscando la razón de ser de los hechos, aunque no supiese el profundo significado de ese proceso. Recuerda que conocía hasta los mínimos detalles del jardín de su casa: los plátanos, el árbol de pan, el viento soplando fuerte, el canto de los pajaritos, las sombras ondulantes de las hojas de los plátanos, entre otros tantos saberes.

En los intercambios entre educadores de jóvenes y adultos en los talleres realizados nos interesa trabajar, de manera particular, la relación entre conocimiento cotidiano y conocimiento escolar. Observamos que emergen tensiones entre la heterogeneidad de los estudiantes, sus saberes, edades, expectativas en relación con la educación y la homogeneidad con la que se implementa muchas veces la propuesta curricular para la modalidad. En aulas de nivel primario de jóvenes y adultos se encuentran en un mismo espacio y tiempo sujetos que no leen ni escriben convencionalmente, mujeres que sienten la necesidad de finalizar ese tramo escolar, jóvenes que fueron expulsados de la escuela en su etapa infantil. Las preguntas de los educadores se centran en los modos de articular contenidos y actividades para dar respuestas a las distintas demandas.

En esa diversidad, es común escuchar "hay que partir de lo que los alumnos saben". Nos preguntamos: ¿y después qué decisiones tomamos?, ¿desde dónde los abordamos?, 
¿cómo trabajamos los objetos de conocimiento de cada área? Es necesario partir de la realidad concreta y no de cuestiones que no sean significativas para los jóvenes y adultos, pero esto implica aportar otros conocimientos integrados en diferentes disciplinas que propongan elementos para interpretar la realidad vivida.

En esa línea, se observa también cierta construcción de sentidos que los docentes atribuyen no solo a lo que propone el currículum como conocimientos a ser transmitidos, sino y de forma singular, a lo que los maestros consideran que los otros, los jóvenes y adultos, "tienen que saber" como sujetos sociales. Por ejemplo, en distintos talleres trabajamos la organización de una propuesta de enseñanza correspondiente al área de Ciencias con el objetivo de vincular el concepto de salud con el de alimentación, por ser considerado como un conocimiento "útil" (en el sentido de uso) para la cotidianeidad de los estudiantes. En un grupo de educadores se decide trabajar "una propuesta para modificar hábitos alimentarios que no resultan saludables", para lo cual se propuso a los estudiantes completar una tabla con los alimentos consumidos en las cuatro comidas diarias durante una semana. En el taller, se pusieron en común las actividades de los estudiantes y se observó la ausencia de información en las celdas correspondientes a la cena o el almuerzo que eran graficadas a partir de una línea. Si bien los educadores identificaron las condiciones de vida de los jóvenes y adultos en la imposibilidad de costear esas comidas, la mayor preocupación estuvo centrada en la ingesta excesiva de harinas e hidratos de carbono por parte de los jóvenes y adultos y en la necesidad de "enseñarles a comer bien" a partir de la pirámide alimentaria.

Nos interesa compartir este ejemplo porque nos interpela como formadores de educadores en el campo de la educación de jóvenes y adultos. Se observa una negación de la situación en la que viven los estudiantes, se asimila la problemática a un desconocimiento de los modos del buen comer sin considerar las reales condiciones de existencia de ese grupo social. Nuevamente las condiciones concretas de existencia se naturalizan y se expresan en un "no reconocimiento" de los saberes que impregnan las decisiones que toman algunos educadores respecto del conocimiento en las aulas de la modalidad.

En Cartas a Cristina. Reflexiones sobre mi vida y mi trabajo (1996), Freire sostiene que un punto de partida en la práctica "es el respeto a la identidad cultural de los educandos, del sesgo de clase que marca tal identidad: vale decir, el respeto por el lenguaje de los educandos, su sintaxis, su prosodia, su semántica y por los conocimientos de experiencia hechos con que llegan a la escuela" (1996: 146).

Si bien en los últimos años la normativa nacional ha generado la elaboración de propuestas curriculares en las distintas jurisdicciones, ${ }^{4}$ se observan tensiones en la construcción de mediaciones entre lo propuesto a nivel ministerial y las prácticas de los docentes de la modalidad. Las escasas ofertas de formación que atienden la especificidad pedagógica articulada con formas de lo escolar que invisibilizan a los sujetos destinatarios han producido sesgos en cuanto a la construcción de saberes y conocimientos. Entendemos que, tras la frase "partir de lo que saben", prevalece una mirada sobre lo que los educadores creen que necesitan los estudiantes y desde allí operan una serie de supuestos que configuran la construcción de la propuesta educativa. Son estos supuestos los que hay que develar junto a ellos en procesos de formación docente.

4 La resolución CFE 118/2010 propone una organización curricular a partir de núcleos conceptuales para los centros educativos de jóvenes y adultos. En distintos desarrollos curriculares de la modalidad producidos en las jurisdicciones de Tucumán, Mendoza, Jujuy y Corrientes, se organiza la propuesta a partir de contextos problematizadores, situaciones problemáticas y núcleos conceptuales en el marco de la normativa nacional y provincial pertinente. 


\section{La tarea político-pedagógica de los educadores: enseñar desde núcleos conceptuales}

Los planteos expuestos en los apartados anteriores nos posicionan en la centralidad de la tarea político-pedagógica de los educadores que se materializa en las formas de organización de la propuesta educativa. En ella se expresan decisiones vinculadas a la direccionalidad del acto educativo, la comprensión del conocimiento y de los sujetos. Particularmente se manifiesta en lo que Freire denomina "situación educativa".

En El grito manso (2014), Freire presenta los elementos constitutivos de una situación educativa. Reconoce, la presencia de un "sujeto", el educador o la educadora, con una tarea específica, la tarea de educar; de los educandos; y del espacio donde estos sujetos se encuentran, al que llama espacio pedagógico. Este espacio también reconoce un tiempo, el "tiempo pedagógico". Freire enfatiza este elemento, sostiene que el tiempo educativo está al servicio de la producción del saber y esto está ligado a ideales. Otro elemento lo constituyen los contenidos curriculares, los "objetos cognoscibles" que "son percibidos mediante el ejercicio de la curiosidad" (2014: 46). Estos elementos constitutivos tienen además "objetivos", que en filosofía de la educación se denominan direccionalidad de la educación. Es la "direccionalidad" la que explica una cualidad que Freire identifica como esencial de la práctica educativa, a la que llama "politicidad de la educación" (2014: 49). Se asocia la politicidad con otras características de la situación educativa: sostiene que en la misma "estética y ética van de la mano".

En nuestro trabajo con educadores, consideramos la situación educativa tomando como metáfora un triángulo, cuyos vértices son sujetos, conocimientos y concientización. Este triángulo se encuentra en un contexto que lo constituyen el espacio y el tiempo pedagógico, a su vez configurado por el contexto social y político. Incorporamos el concepto de concientización porque entendemos que se trata de un proceso de objetivación de la realidad, que implica el conocimiento crítico de la misma para transformarla, en el sentido dialéctico que Freire establece en La importancia de leer y el proceso de liberación (2001b). Señalamos que la concientización no se da en el vacío ni es un resultado lineal, sino que es un proceso relacionado a un contexto histórico social o situado (tiempo y espacio) de los sujetos (estudiantes y educadores).

A partir de este análisis, entendemos que no se trata de técnicas de enseñanza sino de un modo de abordar los conocimientos, tanto cotidianos como curriculares, que tiene como sustento una teoría que posiciona al sujeto como sujeto creador con conocimiento y a la educación como acto político. Nuestra propuesta es la elaboración de núcleos conceptuales como una construcción pedagógica enmarcada en una direccionalidad política. Un núcleo conceptual es una forma de organizar los conocimientos y la propuesta de enseñanza a partir de conceptos básicos dentro de una estructura teórico-metodológica que permite abordar, discutir, profundizar una problemática en particular desde las distintas disciplinas. Se trata de nociones básicas que posibilitan la relación entre las distintas áreas.

Básicas en el sentido de conocimiento de uso, aquel que le permite al sujeto poder cuestionarse sobre el por qué, el para qué de los hechos o fenómenos y le brinde la posibilidad de relacionar los procesos inmediatos y mediatos; de ninguna manera avalamos el concepto de contenidos mínimos en el sentido de elementales. (Lorenzatti, 2005: 40)

Esta manera de concebir los procesos de enseñanza le otorga, por un lado, centralidad a las decisiones del docente en relación con el conocimiento y a la directividad de la práctica educativa. Es decir, las decisiones no se toman en el vacío ya que la intervención es "histórica, cultural y política" (Freire, 2001b: 53). Por el otro lado, jerarquiza la palabra del estudiante sobre su "saber propio de la experiencia vivida" al integrarlo en la construcción de la propuesta. 
Desde esa perspectiva, en nuestra propuesta pedagógica sugerimos a los educadores realizar un análisis del contexto (sociopolítico, económico e institucional) donde se desarrolla la experiencia educativa, e identificar/construir la situación problemática que se expresa en un enunciado del núcleo conceptual de manera propositiva o interrogativa. En esta formulación hay una decisión e intencionalidad de focalizar en determinadas prácticas sociales y analizar sus vinculaciones conceptuales, teniendo en cuenta los principios y contenidos curriculares de la modalidad. No es una tarea sencilla de simple definición. Muchas veces la misma refleja la visión de los docentes más allá de la percepción que los estudiantes tienen del problema. Son los sujetos jóvenes y adultos quienes pueden desvelar las situaciones problemáticas de su entorno inmediato a partir de sus conocimientos cotidianos. Por esta razón, se requiere una construcción conjunta para identificar sus reales necesidades y saber qué es lo que ellos identifican como una situación problemática. Es decir, que la particular mirada de los estudiantes sobre sus necesidades y la de su contexto deben formar parte de la construcción del fenómeno que se propone estudiar (Freire, 2002a).

En La importancia de leer y el proceso de liberación, Freire sostiene que el conocimiento de la realidad es indispensable para el desarrollo de la conciencia de sí. Agrega también que el acto de conocer exige el desvelamiento de su objeto, que no se da en la dicotomía entre subjetividad y objetividad, acción y reflexión, práctica y teoría. Destaca la importancia de que "(...) en la práctica del desvelamiento de la realidad, en el proceso concientizador, que la realidad sea aprehendida no como algo que es, sino como devenir, como algo que está siendo" (2001b: 85).

En tal sentido, la potencialidad de los núcleos reside en la red conceptual que construyen los docentes en función de los intereses y las condiciones de vida de los estudiantes. No solo se trata de conceptos básicos, sino también de una estructura teórico-metodológica que permite abordar, discutir, profundizar una problemática en particular desde las distintas disciplinas. Necesariamente su construcción implica una reinterpretación y recreación de los contenidos que propone el diseño curricular de la modalidad.

Es el educador quien decide, en base a esta situación y a los objetivos planteados, cuáles son los conceptos y contenidos disciplinares, las actividades y los textos que ofrecen herramientas para que los sujetos puedan objetivar esa realidad y tener una comprensión crítica de la misma. Entendidas las prácticas de enseñanza de esta manera, el docente se constituye en un mediador entre los conocimientos cotidianos de los jóvenes y adultos y los conocimientos escolares. Por esta razón, se hace necesario focalizar en sus decisiones en torno a la práctica pedagógica y los sentidos otorgados a la organización de la enseñanza para favorecer un tránsito desde una concepción digestiva del conocimiento a un conocimiento explicativo de la realidad. Dice Freire en Pedagogía del Oprimido que "...el papel del educador problematizador es el de proporcionar, conjuntamente con los educandos, las condiciones para que se dé la superación del conocimiento al nivel de la 'doxa' por el conocimiento verdadero, el que se da al nivel del 'logos"' (2000: 88).

En trabajos anteriores (Lorenzatti, 2005; Lorenzatti et al., 2019) explicitamos las decisiones que los educadores pueden tomar en el proceso de construcción de los núcleos conceptuales, y no hacerlo de un modo instrumental sino orientativo en su accionar cotidiano en la selección de conceptos que delimitan y explican la situación problemática desde las distintas disciplinas; en una jerarquización entre los conocimientos a enseñar; y en la propuesta de actividades con raíz en las experiencias vivenciales de los estudiantes para generar procesos de real apropiación de los mismos. Estas actividades de aprendizaje implican el trabajo con distintos tipos de textos, evidenciando decisiones de los docentes en donde profundizar en función de las características e intereses de los estudiantes. 
Este modo de abordar la enseñanza en experiencias educativas con jóvenes y adultos requiere procesos de formación específica donde se discuta con los educadores sobre ciertas disposiciones básicas para trabajar. Freire en Pedagogía de la autonomía (1997) habla de los saberes necesarios para la práctica pedagógica y las condiciones en las que un educador debe trabajar para orientar en la construcción de sujetos creadores y autónomos. Sostiene que enseñar exige rigurosidad metódica, investigación, criticidad, estética y ética, uso de las palabras a través del ejemplo, humildad, tolerancia y lucha en defensa de los derechos de los educadores, convicción de que el cambio es posible y, por lo tanto, alegría y esperanza. También destaca que enseñar implica aceptar lo nuevo y rechazar todo tipo de discriminación. En relación con los estudiantes, Freire sostiene que enseñar exige respeto a los saberes de los educandos, reconocimiento de la asunción de la identidad cultural, conciencia de inacabamiento y, en esta línea, reconocimiento de ser sujeto condicionado y respeto hacia la autonomía de los educandos.

En todos sus libros Freire habla de la curiosidad asociada al conocimiento. De manera particular, en educación de jóvenes y adultos esta premisa implica un fuerte trabajo por parte de los educadores en el desarrollo de la capacidad crítica de "tomar distancia" del objeto, de observarlo, de delimitarlo, de acercar el objeto o hacer su aproximación metódica, su capacidad de comparar, de preguntar. En la Novena Carta (2002b: 128), Freire remite al contexto teórico, el de la formación permanente de los educadores, y señala que es indispensable "la reflexión crítica sobre los condicionamientos que el contexto cultural ejerce sobre nosotros, sobre nuestro modo de actuar, sobre nuestros valores" (ibídem). Insiste en que el contexto teórico, formador, "jamás puede transformarse en un contexto del puro hacer, como a veces se piensa ingenuamente. Al contrario, es el contexto del quehacer, de la praxis, vale decir, de la práctica y de la teoría. La dialéctica entre la práctica y la teoría debe ser plenamente vivida" (ibídem: 129). En dicho marco, este proceso abre a la circulación de las preguntas, a la reflexión crítica sobre la propia pregunta y estimula la creatividad y la imaginación desde una perspectiva dialógica.

\section{Reflexiones finales}

A lo largo de este artículo nos propusimos compartir algunas reflexiones sobre el lugar del conocimiento en el mundo adulto, a la luz de los desarrollos teóricos de Paulo Freire. Queda aún mucho por analizar de su obra para orientar los procesos de enseñanza en este campo socioeducativo.

Un punto de partida que enriquecerá las discusiones sobre la enseñanza en educación de jóvenes y adultos radica en reconocer a los sujetos, tanto estudiantes como docentes, como sujetos de derechos desde sus potencialidades y no desde sus carencias. Saleme (1997) señala la importancia de mirar al adulto concreto, que vive en determinadas condiciones sociales, y no a un sujeto abstracto que se constituye en objeto de estudio de los organismos internacionales. Hablamos del joven o adulto con conocimientos de base empírica, pragmática porque "la construcción del conocimiento en estos sectores tiene y mantiene una dinámica vital, porque el objeto no está afuera, ajeno, para ser conocido; está en el sujeto, con el sujeto, actuando conjuntamente en lo cotidiano" (Saleme, 1997: 138).

En esta línea, reconocemos a otro sujeto, el educador que también está en proceso de lucha, frente a la denigración de su tarea docente a lo largo de años de neoliberalismo. Se hace necesario recuperar junto a ellos los saberes pedagógicos que fueron construyendo en su práctica cotidiana, fortalecer la confianza en su quehacer pedagógico cotidiano para consolidar procesos de aprendizaje en su práctica docente; propiciar reflexiones acerca de sus intencionalidades para posicionarse como orientador del proceso de 
aprendizaje de los estudiantes. Insistimos en señalar que el educador no es un mero ejecutor de prescripciones curriculares, sino que va tejiendo una red de contenidos que ofrecen una organización de relaciones de conocimientos disciplinares con el objetivo de ayudar al joven y al adulto a interpretar la realidad en la que viven.

En este artículo compartimos una propuesta de trabajo con el conocimiento que iniciamos en la década de 1990 junto a María Saleme. Hablamos de la construcción de núcleos conceptuales que superan la visión de un listado de contenidos porque se trata del desarrollo de un proceso de mediación que ubica el conocimiento a enseñar y los modos de hacerlo en función de una situación problemática sobre los sujetos sociales, sus identidades y su constitución como sujetos en el barrio. No hay una decisión a priori de los conceptos centrales a definir. Siempre estará en relación con los grupos de jóvenes y adultos que asisten al espacio educativo y al vínculo pedagógico que allí se construye.

Desde esta propuesta, valorizamos los procesos de decisión de los educadores sobre su propia práctica para permitir el desarrollo de una enseñanza que posibilite la apropiación de conocimientos por parte de los estudiantes; que promueva la auténtica relación entre curiosidad, análisis de la realidad, construcción de conocimientos y toma de conciencia sobre el lugar que cada uno ocupa en la sociedad.

En síntesis, entendemos de acuerdo con lo postulado por Freire en El grito manso que

(...) no hay práctica educativa sin sujetos, sin sujeto educador y sin sujeto educando; no hay práctica educativa fuera de ese espacio-tiempo que es el espacio-tiempo pedagógico; no hay práctica educativa fuera de la experiencia de conocer que técnicamente llamamos experiencia gnoseológica, que es la experiencia del proceso de producción del conocimiento en sí; no hay práctica educativa que no sea política; no hay práctica educativa que no esté envuelta en sueños; no hay práctica educativa que no involucre valores, proyectos, utopías, no hay, entonces práctica educativa sin ética. (2014: 50-51)

Desde nuestro lugar de docentes de una universidad pública consideramos que la obra de Freire nos estimula a seguir trabajando en investigación, docencia y extensión porque es la manera de construir nuevos conocimientos con la participación de los sujetos del campo de la educación de jóvenes y adultos. 


\section{Q Bibliografía}

" Bowman, M. A. (2011). Actividades de lengua escrita con jóvenes de baja escolaridad: un análisis desde la mediación docente. En Lorenzatti, M. del C. (comp.). Procesos de alfabetización y acceso a la educación básica de jóvenes y adultos, pp. 137-159. Córdoba, Vaca Narvaja.

"Dichiara, M. B. (2018). “Estudiar: una trama compleja de jóvenes y adultos”. Tesis de Licenciatura en Ciencias de la Educación. Córdoba, Escuela de Ciencias de la Educación, FFyH, UNC.

»Freire, P. (1975) Acción cultural para la libertad. Buenos Aires, Tierra Nueva.

»Freire, P. (1976). La educación como práctica de la libertad, 4ª ed, Bueos Aires, Siglo XXI.

»Freire, P. (1996). Cartas a Cristina. Reflexiones sobre mi vida y mi trabajo. México, Siglo XXI.

"Freire, P. (1997). Pedagogía da autonomía. Saberes necesarios a practica educativa. San Pablo, Paz e Terra. Colección Leitura.

》Freire, P. (2000). Pedagogía del Oprimido, 53ํe ed. Guadalajara, Siglo XXI.

»Freire, P. (2001a). Política y educación, $5^{2}$ ed. México, Siglo XXI.

»Freire, P. (2001b). La importancia de leer y el proceso de liberación, 14ํำ ed. México, Siglo XXI.

»Freire, P. (2002a). Pedagogía de la esperanza. Un reencuentro con Pedagogía del Oprimido, $5^{\circ}$ ed. México, Siglo XXI

》Freire, P. (2002b). Cartas a quien pretende enseñar. Buenos Aires, Siglo XXI.

»Freire, P. (2014). El grito manso, $3^{\circ}$ ed. Buenos Aires, Siglo XXI.

»Heller, A. (1977). Sociología de la vida cotidiana. Barcelona, Península.

"Kalman, J. (2004). Saber lo que es la letra. Una experiencia de la lectoescritura con las mujeres de Mixquic. México, Siglo XXI.

» Kalman, J. y Street, B. V. (coords.) (2009). Lectura, escritura y matemáticas como prácticas sociales. Diálogos con América Latina. México, Siglo XXI.

» Kalman, J.; Lorenzatti, M. del C.; Hernández G.; Méndez, A. M. y Blazich, G. (2018). La relevancia de la alfabetización de personas jóvenes y adultas en América Latina hoy. México, Centro de Cooperación Regional de Educación de Adultos de América Latina y el Caribe (CREFAL).

"Lorenzatti, M. del C. (2005). Saberes y conocimientos sobre cultura escrita: un trabajo con maestros de jóvenes y adultos. Córdoba, Ferreyra.

»Lorenzatti, M. del C. (2016). Informe Final. Proyecto "Estudio de la situación de las políticas de formación docente para educación de jóvenes y adultos y educación rural en países del Mercosur". "Programa de Apoyo al Sector Educativo del MERCOSUR (PASEM)". Córdoba, Prosecretaría de Relaciones Internacionales, Universidad Nacional de Córdoba.

»Lorenzatti, M. del C. (2018). Conocimientos, prácticas sociales y usos escolares de cultura escrita de adultos de baja escolaridad. México, Centro de Cooperación Regional de Educación de Adultos de América Latina y el Caribe (CREFAL). 
"Lorenzatti, M. del C. y Arrieta, R. (2017). La escuela de jóvenes y adultos: un espacio para aprender "la charla" y la "buena escritura". En Freitas, A. F. Ribeiro de; Freitas, M. de Lima de Queiroz; Ribeiro; N. y Aguiar, N. (orgs.). Os sentidos da formação em rede: observações sobre as práticas de leitura e das práticas dos leitores. Maceió, Edufal.

» Lorenzatti, M. del C.; Tosolini, M.; Bowman M. A.; Arrieta R.; Delprato M. F. y Montenegro M. (2019). Cuaderno de Trabajo. Notas para la enseñanza en centros educativos de jóvenes y adultos. Municipalidad de Córdoba, Editorial de la Municipalidad de Córdoba, Secretaría de Cultura, Dirección de Patrimonio CulturalUNC.

》 Rodríguez, L. (2015). Paulo Freire. Una biografía intelectual. Surgimiento y maduración de la pedagogía del oprimido. Buenos Aires, Colihue.

"Saleme, M. (1997). Decires. Córdoba, Vaca Narvaja.

» Street, B. (1984). Literacy and theory practice. Cambridge, Cambridge University Press.

"Street, B. (2009). Perspectivas etnográficas y políticas sobre cultura escrita: el poder de nombra y definir. En Kalman, J. y Street, B. V. (coords.). Lectura, escritura y matemáticas como prácticas sociales. Diálogos con América Latina. México, Siglo XXI.

»Zavala, V.; Niño Murcia, M. y Ames, P. (eds.). (2004). Escritura y Sociedad. Nuevas perspectivas teóricas y etnográficas. Lima, Red para el Desarrollo de las Ciencias Sociales en el Perú.

\section{María del Carmen Lorenzatti}

Doctora en Ciencias de la Educación, docente investigadora/Centro de Investigaciones María Saleme de Burnichon, Facultad de Filosofía y Humanidades, Universidad Nacional de Córdoba. Depto. de Ciencias de la Educación y la Salud, Universidad Nacional de Chilecito, Argentina. marieta.lorenzatti@gmail.com

\section{Mariana Tosolini}

Doctora en Estudios Sociales de América Latina, docente investigadora/Centro de Investigaciones María Saleme de Burnichon, Facultad de Filosofía y Humanidades, Universidad Nacional de Córdoba. Depto. de Ciencias de la Educación y la Salud, Universidad Nacional de Chilecito, Argentina. marianatosolini@gmail.com 
